\title{
On the Speiser equivalent for the Riemann hypothesis
}

\author{
Ramūnas Garunkštis • Raivydas Šimènas
}

Received: 5 September 2014 / Revised: 11 November 2014 / Accepted: 14 November 2014 /

Published online: 6 January 2015

(C) Springer International Publishing AG 2015

\begin{abstract}
Speiser showed that the Riemann hypothesis is equivalent to the absence of non-trivial zeros of the derivative of the Riemann zeta-function left of the critical line. We investigate the relationship between the non-trivial zeros of the functions belonging to the extended Selberg class and of their derivatives left of the critical line. Every element of this class satisfies a functional equation of the Riemann type, but it contains zeta-functions for which the Riemann hypothesis is not true. As an example, we study the relationship between the trajectories of zeros of a certain linear combination of Dirichlet $L$-functions and of its derivative computationally. In addition, we examine Speiser type equivalent for Dirichlet $L$-functions with imprimitive characters for which the Riemann hypothesis is not true and which do not satisfy the Riemann type functional equation.
\end{abstract}

Keywords Extended Selberg class - Derivatives of zeta-functions · Zeros of zeta-functions · Dirichlet $L$-functions

Mathematics Subject Classification $11 \mathrm{M} 26 \cdot 11 \mathrm{M} 41$

The first author is supported by Grant No. MIP-066/2012 from the Research Council of Lithuania.

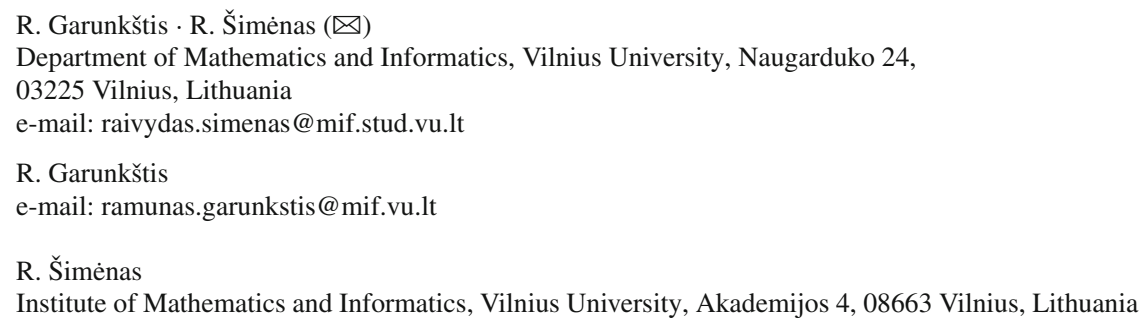




\section{Introduction and statement of results}

In the first part of the 20th century, Speiser [14] studied the relationship between the location of zeros of the derivative of the Riemann zeta-function and the Riemann hypothesis $(\mathrm{RH})$. His result, achieved by geometric means, is that the $\mathrm{RH}$ is equivalent to the absence of non-real zeros of the derivative of the Riemann zeta-function left of the critical line.

Later on, Levinson and Montgomery [9] investigated the relationship between zeros of the Riemann zeta-function and its derivative analytically. They proved the quantitative version of the Speiser's result, namely, that the Riemann zeta-function and its derivative have approximately the same number of zeros left of the critical line. This result was extended to Dirichlet $L$-functions with primitive Dirichlet characters (Yıldırım [20]), to the Selberg class (Šleževičienè [18]), to the Selberg zeta-function on a compact Riemann surface (Luo [10], see also Garunkštis [3,4]). In all these cases, an analog of the RH is expected or, as in the case of the Selberg zeta-function, it is known to be true. Here we ask the question if the Speiser equivalent is valid for zeta-functions for which an analog of the RH is not true. The answer is positive and as an example we consider numerically certain zeta-functions depending on a parameter, which for some values of the parameter violate the RH and for other values they are expected to satisfy the RH. To be more general, we choose to investigate the so called extended Selberg class. This class includes classical zeta-functions, such as the Riemann zeta-function, Dirichlet $L$-functions for primitive characters, Dedekind zeta-functions. This class also contains linear combinations of certain Dirichlet $L$-functions for which the RH is not valid, see Kaczorowski and Kulas [6].

A not identically vanishing Dirichlet series

$$
F(s)=\sum_{n=1}^{\infty} \frac{a_{n}}{n^{s}}
$$

which converges absolutely for $\sigma>1$ belongs to the extended Selberg class $S^{\#}$ if:

- (meromorphic continuation) There exists $k \in \mathbb{N}$ such that $(s-1)^{k} F(s)$ is an entire function of finite order.

- (functional equation) $F(s)$ satisfies the functional equation

$$
\Phi(s)=\omega \overline{\Phi(1-\bar{s})},
$$

where $\Phi(s)=F(s) Q^{s} \prod_{j=1}^{r} \Gamma\left(\lambda_{j} s+\mu_{j}\right)$, with $Q>0, \lambda_{j}>0, \operatorname{Re} \mu_{j} \geq 0$ and $|\omega|=1$.

While the data $Q, \lambda_{j}, \mu_{j}$ and $\omega$ of the functional equation are not uniquely determined by $F$, the value $d_{F}=2 \sum_{j=1}^{r} \lambda_{j}$ is an invariant. It is called the degree of $F$. More about the (extended) Selberg class see the survey papers of Kaczorowski and Perelli [5, $7,8,11,12]$.

For a positive degree, the zeros of $F(s)$ located at poles of the Gamma functions in functional equation (1), i.e. at $s=-\left(\mu_{j}+k\right) / \lambda_{j}$ with $k=0,1,2, \ldots$ and $j=$ 
$1, \ldots, r$, are called trivial zeros. If the degree is equal to zero, then the functional equation of $F(s)$ has no Gamma factors and thus $F(s)$ has no trivial zeros. For any degree, let $\sigma_{F} \geq 1 / 2$ be the least real number such that $F(s)$ does not have any zeros in the right-half plane $\sigma>\sigma_{F}$, cf. Kaczorowski [5, Section 2.1]. Then by the functional equation (1) we see that in the left-half plane $\sigma<1-\sigma_{F}$ the function $F(s)$ can have only trivial zeros.

Suppose that $F(s) \in S^{\#}$ is a non-constant function. Next, we consider zero free regions of $F^{\prime}(s)$ in the left-half plane. If the degree is equal to zero, then $F(s)$ is a Dirichlet polynomial whose structure is well understood, see Kaczorowski and Perelli [7] or formula (10) below. It follows that for $d_{F}=0$, there is $\sigma_{1}$ such that $F^{\prime}(s) \neq 0$ if $\sigma \leq \sigma_{1}$. For $d_{F}>0$, a zero free region of $F^{\prime}(s)$ is described by the next proposition.

Proposition 1.1 Let $F(s) \in S^{\#}$ and $d_{F}>0$. Then there is $\tau \geq 0$ such that $F^{\prime}(s) \neq 0$ in $\sigma<1-\sigma_{F},|t| \geq \tau$.

From the proof of Proposition 1.1, we see that for a given function $F(s)$ the explicit upper bound for $\tau$ can be calculated.

In this paper, $T$ always tends to plus infinity. The main results of this article for the functions of the extended Selberg class are the following.

Theorem 1.2 Let $F(s) \in S^{\#}, d_{F}>0$ and $\sigma_{0}>\sigma_{F}$. Let $\tau$ be the same as in Proposition 1.1. Let $N(T)$ and $N_{1}(T)$ respectively denote the number of zeros of $F(s)$ and $F^{\prime}(s)$ in the region $\tau<t<T, \sigma<1 / 2$. Then

$$
N(T)=N_{1}(T)+O(\log T) .
$$

Moreover, if $N(T)<T /\left(2 \sigma_{0}-1\right)+O(1)$, then there is a monotonic sequence $\left\{T_{j}\right\}$, $T_{j} \rightarrow \infty, j \rightarrow \infty$ such that

$$
N\left(T_{j}\right)-N\left(T_{1}\right)=N_{1}\left(T_{j}\right)-N_{1}\left(T_{1}\right) .
$$

Theorem 1.3 Let $F(s) \in S^{\#}$ be a non-constant function with $d_{F}=0$. Let $N(T)$ and $N_{1}(T)$ respectively denote the number of zeros of $F(s)$ and $F^{\prime}(s)$ in the region $0<t<T, \sigma<1 / 2$. Then

$$
N(T)=N_{1}(T)+O(1)
$$

It is well known that $\zeta^{\prime}(1 / 2+i t) \neq 0$ if $\zeta(1 / 2+i t) \neq 0$, see Spira [15, Corollary 3]. Analogous statement is true for the functions from the extended Selberg class.

Proposition 1.4 Let $F(s) \in S^{\#}$ be a non-constant function. Then there is $\tau \geq 0$ such that, for $t \geq \tau$,

$$
F^{\prime}\left(\frac{1}{2}+i t\right) \neq 0 \quad \text { if } \quad F\left(\frac{1}{2}+i t\right) \neq 0
$$

Moreover, if $d_{F}=0$ then $\tau=0$. 
As we already mentioned, the extended Selberg class contains zeta-functions for which the RH is not true, but all these functions still satisfy functional equations (1) of the Riemann type. Can we extend the Speiser equivalent to zeta-functions without such functional equations? Possibly the simplest example of such functions are Dirichlet $L$-functions with imprimitive characters.

For a Dirichlet character $\chi$, the Dirichlet $L$-function $L(s, \chi)$ is defined by

$$
L(s, \chi)=\sum_{n=1}^{\infty} \frac{\chi(n)}{n^{s}} .
$$

Now, suppose $\chi$ is an imprimitive Dirichlet character modulo $q$ induced by a primitive Dirichlet character $\chi_{1} \bmod q_{1}$. Then $q_{1}$ divides $q$ and

$$
\chi(n)= \begin{cases}\chi_{1}(n) & \text { if } \operatorname{gcd}(n, q)=1 \\ 0 & \text { otherwise }\end{cases}
$$

The following Euler product holds:

$$
L(s, \chi)=L\left(s, \chi_{1}\right) \prod_{p \mid q}\left(1-\chi_{1}(p) p^{-s}\right) .
$$

We see that $L(s, \chi)$ has zeros on the line $\sigma=0$ and in this sense the RH is not valid. Also, a functional equation of the Riemann type does not hold for $L(s, \chi)$.

In analogy with Proposition 1.1, we demonstrate the existence of a zero-free region in the left half-plane for $L^{\prime}(s, \chi)$.

Proposition 1.5 Let $\chi$ be an imprimitive Dirichlet character. Then for any $\sigma_{0}>1$, there exists $\tau$ such that $L^{\prime}(s, \chi)$ does not vanish for $\sigma \leq 1-\sigma_{0}$ and $|t| \geq \tau$.

The next theorem is analogous to Theorem 1.2.

Theorem 1.6 Let $\chi \bmod q$ be an imprimitive Dirichlet character induced by a primitive character $\chi_{1} \bmod q_{1}$. Let $\tau$ be the same as in Proposition 1.5. Let $N(T)$ and $N_{1}(T)$ denote the number of zeros of respectively $L(s, \chi)$ and $L^{\prime}(s, \chi)$ in the region $\tau<t<T, \sigma<1 / 2$. Then

$$
N_{1}(T)=N(T)+O(\log T) \geq \frac{\log \left(q / q_{1}\right)}{2 \pi} T+O(\log T),
$$

where the inequality can be replaced by the equality provided the Riemann hypothesis is valid for $L\left(s, \chi_{1}\right)$.

From the proof we see that Theorem 1.6 can be generalized to zeta-functions from the extended Selberg class, multiplied by some simple function. On the other hand, Hurwitz and Lerch zeta-functions also do not satisfy the Riemann type functional equation, but computations do not confirm the Speiser equivalent in these cases. The proofs of above results follow the proof of Levinson and Montgomery [9]. 
The next section is devoted to computer calculations. To illustrate Theorem 1.2 "in action," we compute the trajectories of zeros of a certain zeta-function depending on a parameter as well as the trajectories of zeros of its derivative. We also calculate several zeros of the derivative of the celebrated Davenport-Heilbronn zeta-function, which is an element of the extended Selberg class and has non-real zeros off the critical line. Sect. 3 contains the proofs.

\section{Computations}

All computations in this section were done using the program Mathematica. Computations should be regarded as heuristic because their accuracy was not controlled explicitly. We investigate the function of the following form:

$$
f(s, \tau)=f_{0}(s) \cdot(1-\tau)+f_{1}(s) \cdot \tau,
$$

where $\tau \in[0,1], f_{0}(s)=\left(1+\sqrt{5} / 5^{s}\right) \zeta(s), \zeta(s)$ is the Riemann zeta-function, and $f_{1}(s)=L(s, \psi)$, where $\psi \bmod 5, \psi(2)=-1$.

For any $\tau$, the function $f(s, \tau)$ satisfies the functional equation

$$
f(s)=5^{-s+1 / 2} 2(2 \pi)^{s-1} \Gamma(1-s) \sin \frac{\pi s}{2} f(1-s)
$$

since both $f_{0}$ and $f_{1}$ satisfy (3). Thus, for any $\tau$, the function $f(s, \tau)$ is an element of the extended Selberg class of degree $d_{f}=1$ and Theorem 1.2 for $f(s, \tau)$ is valid.

It is quite likely that all non-trivial zeros of $f(s, 0)$ and $f(s, 1)$ are located on the line $\sigma=1 / 2$. However, by the joint universality theorem for Dirichlet $L$-functions, it follows that for any $0<\tau<1$ there are infinitely many zeros of $f(s, \tau)$ in the strip $1 / 2<\operatorname{Re} s<1$, see Kaczorowski and Kulas [6, Theorem 2].

By $f_{s}^{(v)}(s, \tau)$ we denote that $v$ th partial derivative of $f$ with respect to $s$,

$$
f_{s}^{(v)}(s, \tau)=\frac{\partial^{v}}{\partial s^{v}} f(s, \tau)
$$

Suppose that $\rho=\rho\left(\tau_{0}\right)$ is a zero of multiplicity $m$ of $f\left(s, \tau_{0}\right)$ (i.e. $f_{s}^{(v)}\left(\rho\left(\tau_{0}\right), \tau_{0}\right)=0$, $\left.v=0,1, \ldots, m-1, f_{s}^{(m)}\left(\rho\left(\tau_{0}\right), \tau_{0}\right) \neq 0\right)$. By Rouché's theorem, we have that for every sufficiently small open disc $D$ with center at $\rho$ in which the function $f\left(s, \tau_{0}\right)$ has no other zeros except for $\rho$, there exists $\delta=\delta(D)>0$ such that each function $f(s, \tau)$, where $\tau \in\left(\tau_{0}-\delta, \tau_{0}+\delta\right)$, has exactly $m$ zeros (counted with multiplicities) in the disc $D$, cf. Balanzario and Sánchez-Ortiz [1, Theorem 1] and Dubickas et al. [2, Lemma 8]. If zero $\rho$ is of multiplicity $m=1$, then there exists a neighborhood of $\tau_{0}$ and some function $\rho=\rho(\tau)$, which is continuous at $\tau_{0}$ and, in addition, satisfies the relation $f(\rho(\tau), \tau)=0$. This way, we can speak about the continuous zero trajectory $\rho(\tau)$. Similarly, the trajectories of zeros of the derivative $f_{S}^{\prime}(s, \tau)$ are understood.

In view of functional equation (3), for any $\tau$, non-real zeros of $f(s, \tau)$ are symmetrically distributed with respect to the critical line $\sigma=1 / 2$. It follows that provided 


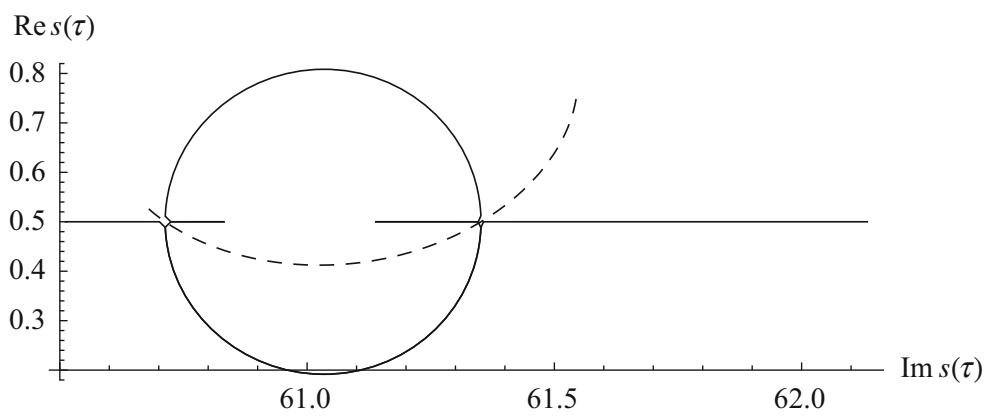

Fig. 1 Solid black trajectories are zero trajectories $\rho_{1}(\tau)$ and $\rho_{2}(\tau): f\left(\rho_{1}(\tau), \tau\right)=0, \rho_{1}(0)=0.5+$ $i 60.84, \rho_{1}(1)=0.5+i 62.13$ and $f\left(\rho_{2}(\tau), \tau\right)=0, \rho_{2}(0)=0.5+i 60.51, \rho_{2}(1)=0.5+i 61.14$. Dashed trajectory is a derivative zero trajectory $q(\tau): f_{S}^{\prime}(q(\tau), \tau)=0, q(0)=0.52+i 60.68, q(1)=0.76+i 61.55$

the RH holds for $f(s, 0)$ and if we have its simple zero $\rho_{1}(0)$, the functional equation forbids the corresponding zero trajectory $\rho_{1}(\tau)$ from leaving the critical line as $\tau$ increases unless it meets the trajectory $\rho_{2}(\tau)$ of another zero, say at $\tau=\tau^{\prime}$. Then $\rho_{1}\left(\tau^{\prime}\right)=\rho_{2}\left(\tau^{\prime}\right)$ is a double zero of $f\left(s, \tau^{\prime}\right)$. In the case of zeros of degree two, they can leave the critical line. Moreover, their trajectories are symmetric with respect to the critical line. Let the trajectories $\rho_{1}(\tau)$ and $\rho_{2}(\tau)$ meet each other at $\tau=\tau^{\prime}$ and let them split into two trajectories, which leave the critical line and which are symmetric with respect to this line. For definitiveness, we say that the trajectory $\rho_{1}(\tau)$ (similarly $\left.\rho_{2}(\tau)\right)$ at $\tau=\tau^{\prime}$ turns to the right hand-side as $\tau$ increases. Then the trajectories $\rho_{1}(\tau)$ and $\rho_{2}(\tau)$ remain defined in the a neighborhood of $\tau^{\prime}$. Note that this definition makes sense because in all calculations in this section, the trajectories $\rho_{1}(\tau)$ and $\rho_{2}(\tau)$ move in opposite directions before they meet at $\tau=\tau^{\prime}$. In addition, the derivative $f_{s}^{\prime}\left(s, \tau^{\prime}\right)$ must vanish at the meeting point $s=\rho_{1}\left(\tau^{\prime}\right)$. Thus there is a trajectory $q(\tau)$ of a zero of $f_{s}^{\prime}(s, \tau)$ such that $q\left(\tau^{\prime}\right)=\rho_{1}\left(\tau^{\prime}\right)=\rho_{2}\left(\tau^{\prime}\right)$.

Figures 1 and 2 are parametric plots of the trajectories of zeros of $f(s, \tau)$ and its derivative, solid and dotted lines respectively. We see that the trajectory of the derivative crosses the critical line in accordance with Theorem 1.2.

To find the solutions, i.e. the zero trajectories, $\rho(\tau)$ and $q(\tau), 0 \leq \tau \leq 1$, of

$$
f(\rho(\tau), \tau)=0 \quad \text { and } \quad f_{s}^{\prime}(q(\tau), \tau)=0,
$$

we solve the differential equations numerically

$$
\frac{\partial \rho(\tau)}{\partial \tau}=-\frac{\partial f(\rho, \tau) / \partial \tau}{\partial f(\rho, \tau) / \partial \rho} \quad \text { and } \quad \frac{\partial q(\tau)}{\partial \tau}=-\frac{\partial^{2} f(q, \tau) / \partial q \partial \tau}{\partial^{2} f(q, \tau) / \partial q^{2}}
$$

As the initial conditions, some zeros of $f(s, 0)=\left(1+\sqrt{5} / 5^{s}\right) \zeta(s)$ and $f_{s}^{\prime}(s, 0)$ are used.

In the upper half-plane region with $0<t \leq 1500$ the function $f(s, 0)$ has 1452 zeros, all on the critical line. According to our computations, 286 of these zeros leave the critical line and, except several cases similar to Fig. 2, are similar to Fig. 1. 


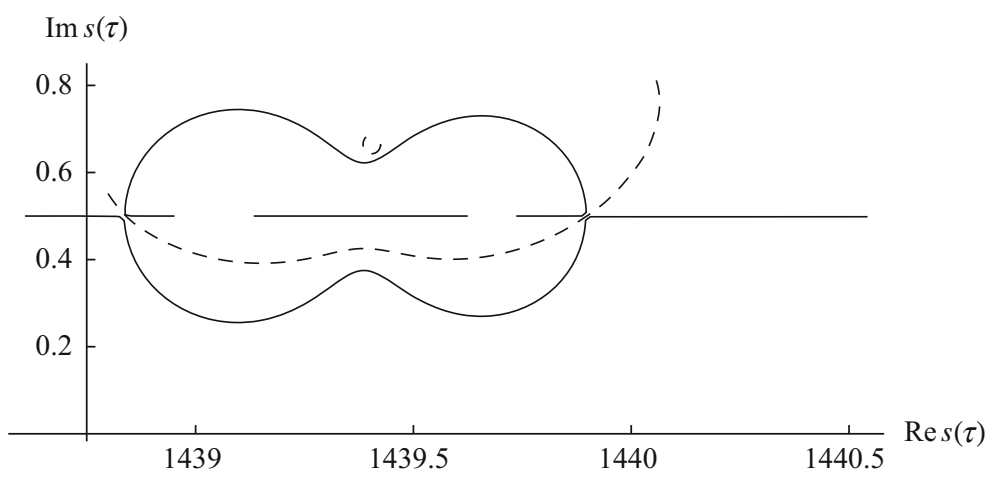

Fig. 2 Solidblack trajectories are zero trajectories with $\rho_{1}(0)=0.50+i 1438.61, \rho_{2}(0)=0.50+i 1438.95$, and $\rho_{3}(0)=0.50+i 1439.62\left(f\left(\rho_{j}(\tau), \tau\right)=0,0 \leq \tau \leq 1, j=1,2,3\right)$. Dashed black trajectories are derivative zero trajectories with $q_{1}(0)=0.55+i 1438.80$, and $q_{2}(0)=0.66+i 1439.42\left(f_{s}^{\prime}\left(q_{j}(\tau), \tau\right)=0\right.$, $0 \leq \tau \leq 1, j=1,2)$

Further, we consider the Davenport-Heilbronn zeta-function defined by

$$
\ell(s)=\frac{1}{2 \cos \alpha}\left(e^{-i \alpha} L(s, \eta)+e^{i \alpha} L(s, \bar{\eta})\right)
$$

where $\eta \bmod 5, \eta(2)=i$, and $\tan \alpha=(\sqrt{10-2 \sqrt{5}}-2) /(\sqrt{5}-1)$. The DavenportHeilbronn zeta-function satisfies the functional equation:

$$
\ell(s)=5^{1 / 2-s} 2(2 \pi)^{s-1} \Gamma(1-s) \cos \frac{\pi s}{2} \ell(1-s) .
$$

The function $\ell(s)$ has zeros with $\sigma>1$ and has infinitely many zeros on the critical line, see Titchmarsh [19, Section 10.25]. Similarly to $f(s, \tau)$, the function $\ell(s)$ has infinitely many zeros in $1 / 2<\sigma<1$. It belongs to the extended Selberg class and hence falls within the class of functions for which Theorem 1.2 holds. It should be noted that zeros of the Davenport-Heilbronn zeta-function have been subject to much analysis. Spira [16] calculated the location of some of zeros of the DavenportHeilbronn zeta-function off the critical line $\sigma=1 / 2$ in the region $0 \leq t \leq 200$, see also Balanzario and Sánchez-Ortiz [1]. He did not find any zeros of its derivative left off $\sigma=1 / 2$ in this region although he did find several locations of zeros of the function itself. This would go against our Theorem 1.2. However, we recalculated zeros of the derivative of the Davenport-Heilbronn zeta-function and we did find zeros left off the critical line with imaginary parts less than 200 .

For $0 \leq t \leq 200$, Spira calculated eight zeros of $\ell(s)$ off the critical line:

$$
0.80+i 85.69, \quad 0.65+i 114.16, \quad 0.57+i 166.47, \quad 0.72+i 176.70
$$


and claimed that 'no zeros of $\ell^{\prime}(s)$ were found in $\sigma<1 / 2,0 \leq t \leq 200$ '. We found four zeros of $\ell^{\prime}(s)$ in $\sigma<1 / 2,0 \leq t \leq 200$ :

$$
0.40+i 85.70, \quad 0.47+i 114.15, \quad 0.49+i 166.47, \quad 0.43+i 176.70 \text {. }
$$

To detect the number of zeros of the function $\ell^{\prime}(s)$ in some region $D$, we compute the integral

$$
\int_{\partial D} \frac{\ell^{\prime \prime}(s)}{\ell^{\prime}(s)} d s,
$$

where $\partial D$ is a boundary of $D$. If there is one zero $q$ in the region $D$, then this zero is computed by the formula

$$
q=\int_{\partial D} s \frac{\ell^{\prime \prime}(s)}{\ell^{\prime}(s)} d s
$$

The zeros were checked using Mathematica's command FindRoot.

\section{Proofs}

Proof of Proposition 1.1 Let $\sigma_{0}>\sigma_{F}$. Also, let $\tau^{\prime}>\tau$ and $\sigma^{\prime}<1-\sigma_{0}$. Let $R$ and $\bar{R}$ be two rectangles with vertices $1-\sigma_{0}+i \tau, 1-\sigma_{0}+i \tau^{\prime}, \sigma^{\prime}+i \tau^{\prime}, \sigma^{\prime}+i \tau$ and $1-\sigma_{0}-i \tau, 1-\sigma_{0}-i \tau^{\prime}, \sigma^{\prime}-i \tau^{\prime}, \sigma^{\prime}-i \tau$ respectively. Using formula (5) below, we will show that there is $\tau$ such that for any $\tau^{\prime}$ and any $\sigma^{\prime}$ the inequality

$$
\operatorname{Re} \frac{F^{\prime}}{F}(s)<0
$$

holds for $s \in R$ and $s \in \bar{R}$. By the argument principle, it follows that $F^{\prime}(s)$ and $F(s)$ have the same number of zeros inside the rectangle $R$ (also in $\bar{R}$ ). This will prove the proposition since for sufficiently large $\tau$, the function $F(s)$ has no zeros inside the rectangles $R$ and $\bar{R}$.

By the definition of the extended Selberg class, there is an integer $n_{F}$ such that the function $G(s)=s^{n_{F}}(s-1)^{n_{F}} \Phi(s)$ is an entire function and $G(1) \neq 0$. By functional equation (1), we have that $G(0) \neq 0$. Moreover, $G(s)$ is an entire function of order 1, see Lemma 3.3 and the comment below the proof of Lemma 3.3 in Smajlović [13]. Applying Hadamard's factorization theorem to the function $G(s)$ analogously as in Šleževičienė [18, Proof of Theorem 3, formula (6)], see also Smajlović [13, formulas (8) and (10)]), we have

$$
\begin{aligned}
\operatorname{Re} \frac{F^{\prime}}{F}(s)=\sum_{\substack{\rho \text { non-trivial } \\
\rho \neq 0,1}} \frac{\sigma-\beta}{|s-\rho|^{2}}-\frac{n_{F} \sigma}{|s|^{2}} & -\frac{n_{F}(\sigma-1)}{|s-1|^{2}}-\log Q \\
& -\operatorname{Re} \sum_{j=1}^{r} \lambda_{j} \frac{\Gamma^{\prime}}{\Gamma}\left(\lambda_{j} s+\mu_{j}\right),
\end{aligned}
$$


where the summation is over the non-trivial zeros of $F(s)$ except possible non-trivial zeros or poles at $s=0$ and $s=1$.

Next we will prove inequality (4). In view of $\Gamma(z) \Gamma(1-z)=\pi / \sin \pi z$, we can write

$$
\sum_{j=1}^{r} \lambda_{j} \frac{\Gamma^{\prime}}{\Gamma}\left(\lambda_{j} s+\mu_{j}\right)=\sum_{j=1}^{r}\left(\lambda_{j} \frac{\Gamma^{\prime}}{\Gamma}\left(1-\lambda_{j} s-\mu_{j}\right)-\lambda_{j} \cot \pi\left(\lambda_{j} s+\mu_{j}\right)\right) .
$$

Recall that $\lambda_{j}>0, j=1, \ldots, r$, and $\sum_{j=1}^{r} \lambda_{j}>0$. Then by the formulas

$$
\begin{aligned}
\frac{\Gamma^{\prime}}{\Gamma}(s) & =\log s+O\left(|s|^{-1}\right), & & \operatorname{Re} s \geq 0, \quad|s| \rightarrow \infty, \\
\cot z & =1+O\left(e^{-|\operatorname{Im} z|}\right), & & \operatorname{Im} z \rightarrow \pm \infty,
\end{aligned}
$$

$\Gamma^{\prime} / \Gamma(s+1)=\Gamma^{\prime} / \Gamma(s)+1 / s$ and equality (5), we have that there is $\tau$ such that, for any $\tau^{\prime}$ and $\sigma^{\prime}$, inequality (4) is true if $s \in R$ and $s \in \bar{R}$. This proves Proposition 1.1.

Proof of Theorem 1.2 Let

$$
R=\left\{s \in \mathbb{C}: \tau<t<T, 1-\sigma_{0}<\sigma<\frac{1}{2}\right\}
$$

To prove the theorem, it is enough to consider the difference of the number of zeros of $F(s)$ and $F^{\prime}(s)$ in the region $R$.

Without loss of generality, we assume that $F(\sigma+i T) \neq 0$ and $F^{\prime}(\sigma+i T) \neq 0$ for $1-\sigma_{0} \leq \sigma \leq 1 / 2$. We consider the change of $\arg F^{\prime} / F(s)$ along the appropriately indented boundary $R^{\prime}$ of the region $R$. More precisely, upper, left, and lower sides of $R^{\prime}$ coincide with the upper, left, and lower boundaries of $R$. To obtain the right-hand side of the contour of $R^{\prime}$, we take the right-hand side boundary of $R$ and deform it to bypass zeros of $F(1 / 2+i t)$ by left semicircles with an arbitrarily small radius.

To prove the first part of Theorem 1.2, we will show that the change of $\arg F^{\prime} / F(s)$ along the contour $R^{\prime}$ is $O(\log T)$. By formula (5), similarly as in the proof of Proposition 1.1, we have

$$
\operatorname{Re} \frac{F^{\prime}}{F}\left(1-\sigma_{0}+i t\right)<0
$$

where $\tau \leq t \leq T$.

We switch to the right-hand side of $R^{\prime}$. For this, we evaluate the terms of equality (5). In view of the symmetry of zeros with respect to the critical line, we consider

$$
\frac{\sigma-\beta}{|s-\rho|^{2}}+\frac{\sigma-1+\beta}{|s-1+\bar{\rho}|^{2}}=-2\left(\frac{1}{2}-\sigma\right) \frac{(t-\gamma)^{2}+(\sigma-1 / 2)^{2}-(1 / 2-\beta)^{2}}{|s-\rho|^{2}|s-1+\bar{\rho}|^{2}}
$$


Let

$$
I_{1}=2 \sum_{\beta<1 / 2} \frac{(t-\gamma)^{2}+(\sigma-1 / 2)^{2}-(1 / 2-\beta)^{2}}{|s-\rho|^{2}|s-1+\bar{\rho}|^{2}}+\sum_{\beta=1 / 2} \frac{1}{|s-\rho|^{2}} .
$$

Then

$$
I=\sum_{\substack{\rho \text { non-trivial } \\ \rho \neq 0,1}} \frac{\sigma-\beta}{|s-\rho|^{2}}=-\left(\frac{1}{2}-\sigma\right) I_{1}
$$

Suppose that $s=1 / 2+i t$ is not a zero of $F(s)$. When $I=0$, see equation (8), then, similarly as in the proof of Proposition 1.1 , we see that $\operatorname{Re} F^{\prime} / F(s)<0$. Let $\rho_{0}=1 / 2+i \gamma_{0}$ be a zero of $F(s)$. Then $I_{1}$, see formula (7), can be made arbitrarily large as we move along the left semicircle with an arbitrarily small radius and center at $\rho_{0}$. This is because the term $1 /\left|s-\rho_{0}\right|^{2} \rightarrow \infty$ as $\left|s-\rho_{0}\right| \rightarrow 0$. Hence on the right-hand side of $R^{\prime}$ we again have $\operatorname{Re} F^{\prime}(s) / F(s)<0$.

By the Phragmén-Lindelöf principle and the functional equation, we obtain that for any $\sigma^{\prime}$ there is $A>0$ such that

$$
F(\sigma+i T)=O\left(T^{A}\right)
$$

uniformly in $\sigma \geq \sigma^{\prime}$, cf. Steuding [17, Theorem 6.8]. By the Cauchy differentiation formula and by the bound (9), we have that the bound analogous to (9) is true also for $F^{\prime}(s)$. Then by using Jensen's theorem, it is possible to show that the change of $\arg F(s)$ and $\arg F^{\prime}(s)$ along the horizontal sides of $R^{\prime}$ is $O(\log T)$, cf. Šležiavičienè [18, Proof of Theorem 1] or Titchmarsh [19, Section 9.4]. This proves the first part of Theorem 1.2.

We will prove the second part of Theorem 1.2. Suppose there is a monotonic sequence $\left\{T_{j}\right\}, T_{j} \rightarrow \infty, j \rightarrow \infty$ with the property $\operatorname{Re}\left(F^{\prime} / F\left(\sigma+i T_{j}\right)\right)<0$, here $1-\sigma_{0}<\sigma<1 / 2$. Then by the first part of the proof we have that $N\left(T_{j}\right)-N\left(T_{1}\right)=$ $N_{1}\left(T_{j}\right)-N_{1}\left(T_{1}\right)$.

Suppose there is no such sequence $\left\{T_{j}\right\}$. Then for sufficiently high $t$, there is $1-\sigma_{0} \leq$ $\sigma \leq 1 / 2$ such that $\operatorname{Re} F^{\prime} / F(s) \geq 0$. Thus $I>0$ and $I_{1}<0$. Then at least one term in $I_{1}$ must be negative. Hence there is a zero $\rho=\beta+i \gamma$ with $1-\sigma_{0}<\beta<1 / 2$ such that

$$
\left(\frac{1}{2}-\beta\right)^{2}>(t-\gamma)^{2}+\left(\sigma-\frac{1}{2}\right)^{2}
$$

It follows that $|t-\gamma|<\sigma_{0}-1 / 2$. Thus, if for sufficiently high $t$ we divide the imaginary line into intervals of length $2 \sigma_{0}-1$, it would follow that for every interval there will be at least one zero whose imaginary part falls into that interval. Since we started with sufficiently high $t$, it follows that in this case $F(s)$ has more than $T /\left(2 \sigma_{0}-1\right)+O(1)$ zeros in the region $R$. This concludes the proof.

Proof of Theorem 1.3 Let us denote the set of degree zero functions of the extended Selberg class by $S_{0}^{\#}$. Let $F(s) \in S_{0}^{\#}$ and let $q=Q^{2}$, where $Q$ is from functional equation (1) of $F(s)$. By Kaczorowski and Perelli [7], we have that $q$ is a positive integer and 


$$
F(s)=\sum_{n \mid q} \frac{a_{n}}{n^{s}},
$$

where

$$
a_{n}=\frac{\omega n}{\sqrt{q}} \overline{a_{q / n}}
$$

moreover, if $\sqrt{q} \in \mathbb{N}$ then $a_{\sqrt{q}}=\varepsilon b$ with $b \in \mathbb{R}$, where $\varepsilon$ denotes a fixed square root of $\omega$. By expression (10), we see that $Q>1$ if $F(s)$ is a non-constant function.

The fact that $d_{F}=0$ means that there are no Gamma factors in the functional equation. Hence

$$
\frac{F^{\prime}}{F}(s)=-2 \log Q-\overline{\frac{F^{\prime}}{F}(1-\bar{s})} .
$$

Let $\sigma_{1}$ be a negative real number with large absolute value such that $F(s) \neq 0$ and $F^{\prime}(s) \neq 0$ for $\sigma \leq \sigma_{1}$, see the comment before Proposition 1.1. Let $R$ be a rectangle with vertices $1 / 2-\delta, 1 / 2-\delta+i T, \sigma_{1}+i T, \sigma_{1}$, where $\delta>0$ is as small as we like and it will be chosen later. Without loss of generality, we assume that $F(s) \neq 0$ and $F^{\prime}(s) \neq 0$ on the boundary of the rectangle $R$. In addition, we assume that $F(s) \neq 0$ and $F^{\prime}(s) \neq 0$ in the interior of the rectangle with vertices $1 / 2,1 / 2+i T, 1 / 2+i T-\delta$, and $1 / 2-\delta$. We can achieve this because zeros of $F$ form a discrete set. To prove the theorem it is enough to show that the change of $\arg F^{\prime} / F(s)$ along the rectangle $R$ is $O(1)$ as $T \rightarrow \infty$.

By formulas (10) and (11), it is easy to see that

$$
\lim _{\sigma \rightarrow-\infty} \frac{F^{\prime}}{F}(\sigma+i t)=-2 \log Q .
$$

Suppose that $s^{\prime}$ is on the left-hand side of $R$ and suppose that $\sigma_{1}$ in the definition of the rectangle $R$ is a negative number with large absolute value. Then $\operatorname{Re} F^{\prime} / F\left(s^{\prime}\right)<0$.

Similarly as in the proof of Theorem 1.2, the change in argument on the horizontal sides is $O(1)$ since $F(s)$ is bounded on vertical strips. We consider the right-hand side $1 / 2-\delta+i t, 0 \leq t \leq T$, of $R$. By equality (11), we see that

$$
\operatorname{Re} \frac{F^{\prime}}{F}\left(\frac{1}{2}+i t\right)=-\log Q
$$

if $1 / 2+i t$ is not a zero of $F(s)$. We claim that there is a sufficiently small $\delta=\delta(T)$ such that, for $0 \leq t \leq T$,

$$
\operatorname{Re} \frac{F^{\prime}}{F}\left(\frac{1}{2}-\delta+i t\right) \leq-\frac{\log Q}{2} .
$$

To prove this inequality, it is enough to consider the case when $t$ is in the neighborhood of a zero $\rho=1 / 2+i \gamma$. We have

$$
\frac{F^{\prime}}{F}(s)=\frac{m}{s-\rho}+m^{\prime}+O(|s-\rho|),
$$


where $m$ is the multiplicity of $\rho$. Hence taking $s=1 / 2-\delta+i t$, we see that

$$
\operatorname{Re} \frac{F^{\prime}}{F}(s)=-\frac{m \delta}{|s-\rho|^{2}}+\operatorname{Re} m^{\prime}+O(|s-\rho|) .
$$

Thus $\operatorname{Re} m^{\prime}=-\log Q$. This proves inequality (12). Therefore, the argument change along the right side of the contour is $O(1)$. This gives the proof of Theorem 1.3.

Proof of Proposition 1.4 Let the degree $d_{F}>0$. Assume the contrary, that there is a large number $t$ such that $F^{\prime}(1 / 2+i t)=0$ and $F(1 / 2+i t) \neq 0$. Then by Hadamard's type formula (5), Gamma function property (6), and using the fact that $\sigma=1 / 2$ in (8), we obtain the contradiction

$$
0=\operatorname{Re} \frac{F^{\prime}}{F}(1 / 2+i t)<0 .
$$

This proves the proposition for $d_{F}>0$. If $d_{F}=0$ then the proposition follows by formula (11).

Now we provide the proofs for our results for the Dirichlet $L$-functions with imprimitive characters.

Proof of Proposition 1.5 Let the character $\chi \bmod q$ be induced by a primitive character $\chi_{1}$. Taking logarithmic derivatives of both sides of equation (2) yields

$$
\frac{L^{\prime}}{L}(s, \chi)=\frac{L^{\prime}}{L}\left(s, \chi_{1}\right)+\sum_{p \mid q} \frac{\chi_{1}(p) p^{-s} \log p}{1-\chi_{1}(p) p^{-s}} .
$$

The sum part on the right hand-side is bounded uniformly for $|\sigma| \geq \delta$ with fixed positive $\delta$. The Dirichlet $L$-function $L\left(s, \chi_{1}\right)$ is an element of the (extended) Selberg class. Thus for $L^{\prime} / L\left(s, \chi_{1}\right)$, equation (5) is valid with $F(s)=L\left(s, \chi_{1}\right)$. By an argument similar to that of Proposition 1.1, we conclude that there exists $\tau$ such that $\operatorname{Re}\left(L^{\prime} / L\left(s, \chi_{1}\right)\right)$ is sufficiently small, that is, negative with large absolute value, to make $\operatorname{Re}\left(L^{\prime} / L(s, \chi)\right)$ negative with $\sigma<1-\sigma_{0},|t| \geq \tau$. Therefore, $L^{\prime}(s, \chi)$ has no zeros in our region, just as $L(s, \chi)$.

Proof of Theorem 1.6 Let $R$ and $R^{\prime}$ be the same as in the proof of Theorem 1.2. Again, without loss of generality, assume $T$ is such that $L(\sigma+i T, \chi)$ and $L^{\prime}(\sigma+i T, \chi)$ do not vanish for $1-\sigma_{0} \leq \sigma \leq 1 / 2$. Let $L\left(s, \chi_{1}\right)$ be the corresponding Dirichlet $L$-function with primitive character $\chi_{1}$. We show that the change of $\arg L^{\prime} / L(s, \chi)$ along the contour of $R^{\prime}$ is $O(\log T)$. 
As to the left boundary of $R^{\prime}$, equation (13) yields $\operatorname{Re}\left(L^{\prime} / L(s, \chi)\right)<0$. Let us look at the right boundary of $R^{\prime}$. Equations (5) and (13) give

$$
\begin{aligned}
\operatorname{Re} \frac{L^{\prime}}{L}(s, \chi)= & \sum_{\substack{\rho \text { non-trivial } \\
\rho \neq 0,1}} \frac{\sigma-\beta}{|s-\rho|^{2}}-\frac{n_{F} \sigma}{|s|^{2}}-\frac{n_{F}(\sigma-1)}{|s-1|^{2}}-\log Q \\
& -\operatorname{Re} \sum_{j=1}^{r} \lambda_{j} \frac{\Gamma^{\prime}}{\Gamma}\left(\lambda_{j} s+\mu_{j}\right)+\operatorname{Re} \sum_{p \mid q} \frac{\chi_{1}(p) p^{-s} \log p}{1-\chi_{1}(p) p^{-s}} .
\end{aligned}
$$

Here the first summation is taken over the non-trivial zeros of $L\left(s, \chi_{1}\right)$. The non-trivial zeros of $L\left(s, \chi_{1}\right)$ occur in symmetric pairs. Let $I_{1}$ and $I$ denote the same magnitudes as in Equations (7) and (8), respectively. Arguing in the same vein as in the proof of Theorem 1.2, we get $L^{\prime} / L(s, \chi)<0$ as $s$ moves along the right countour of $R^{\prime}$ provided we take $\tau$ sufficiently large. As to the remaining estimate of the change in argument of $L^{\prime} / L(s, \chi)$ as we move along the horizontal segments of the contour of $R^{\prime}$, we argue exactly as in the proof of Theorem 1.2, which yields that the change in argument is $O(\log T)$. This gives us the proof of the theorem.

Acknowledgments We would like to thank the anonymous referee for his/her valuable comments and insights.

\section{References}

1. Balanzario, E.P., Sánchez-Ortiz, J.: Zeros of the Davenport-Heilbronn counterexample. Math. Comp. 76(260), 2045-2049 (2007)

2. Dubickas, A., Garunkštis, R., Steuding, J., Steuding, R.: Zeros of the Estermann zeta function. J. Aust. Math. Soc. 94(1), 38-49 (2013)

3. Garunkštis, R.: Note on zeros of the derivative of the Selberg zeta-function. Arch. Math. (Basel) 91(3), 238-246 (2008)

4. Garunkštis, R.: Corrigendum to "Note on zeros of the derivative of the Selbergzeta-function". Arch. Math. (Basel) 93(2), 143-145 (2009)

5. Kaczorowski, J.: Axiomatic theory of $L$-functions: the Selberg class. In: Perelli, A., Viola, C. (eds.) Analytic Number Theory. Lecture Notes in Mathematics, vol. 1891, pp. 133-209. Springer, Berlin (2006)

6. Kaczorowski, J., Kulas, M.: On the non-trivial zeros off the critical line for $L$-functions from the extended Selberg class. Monatsh. Math. 150(3), 217-232 (2007)

7. Kaczorowski, J., Perelli, A.: On the structure of the Selberg class, I: $0 \leq d \leq 1$. Acta Math. 182(2), 207-241 (1999)

8. Kaczorowski, J., Perelli, A.: The Selberg class: a survey. In: Győry, K., Iwaniec, H., Urbanowicz, J. (eds.) Number Theory in Progress, vol. 2, pp. 953-992. De Gruyter, Berlin (1999)

9. Levinson, N., Montgomery, H.L.: Zeros of the derivatives of the Riemann zeta-function. Acta Math. 133, 49-65 (1974)

10. Luo, W.: On zeros of the derivative of the Selberg zeta function. Am. J. Math. 127(5), 1141-1151 (2005)

11. Perelli, A.: A survey of the Selberg class of $L$-functions, part I. Milan J. Math. 73, 19-52 (2005)

12. Perelli, A.: A survey of the Selberg class of $L$-functions, part II. Riv. Mat. Univ. Parma (7), $3^{*} 83-118$ (2004)

13. Smajlović, L.: On Li's criterion for the Riemann hypothesis for the Selberg class. J. Number Theory 130(4), 828-851 (2010)

14. Speiser, A.: Geometrisches zur Riemannschen Zetafunktion. Math. Ann. 110, 514-521 (1935)

15. Spira, R.: On the Riemann zeta function. J. London Math. Soc. s1-44(1), 325-328 (1969) 
16. Spira, R.: Some zeros of the Titchmarsh counterexample. Math. Comp. 63(208), 747-748 (1994)

17. Steuding, J.: Value-Distribution of $L$-Functions. Lecture Notes in Mathematics. Springer, Berlin (2007)

18. Šleževičienè, R.: Speiser's correspondence between the zeros of a function and its derivative in Selberg's class of Dirichlet series. Fiz. Mat. Fak. Moksl. Semin. Darb. 6, 142-153 (2003)

19. Titchmarsh, E.C.: The Theory of the Riemann Zeta-Function, 2nd edn. Clarendon Press/Oxford University Press, New York (1986)

20. Yıldırım, C.Y.: Zeros of derivatives of Dirichlet $L$-functions. Turkish J. Math. 20(4), 521-534 (1996) 